\title{
DETERMINACIÓN DE ENERGÍA METABOLIZABLE DEL ACEITE COMPUESTO Y ACEITE RESIDUAL DE FRITURA EN POLLOS POR EL MÉTODO DE COLECCIÓN TOTAL
}

\author{
DETERMINATION OF METABOLIZABLE ENERGY OF THE COMPOUND OIL \\ AND RESIDUAL FRYING OIL IN CHICKENS BY THE TOTAL COLLECTION METHOD
}

Dantón Jorge Miranda Cabrera ${ }^{1}$, Victor Vergara Rubín ${ }^{2}$

\section{RESUMEN}

Objetivo: Determinar la energía metabolizable en pollos de los insumos aceite compuesto y aceite residual de frituras, mediante el método de colección total. Material y Métodos: Se empleó tres tipos de dietas: dieta referencial, dieta con el insumo aceite compuesto y dieta con el insumo aceite residual de frituras; cada dieta fue probada en 10 pollos con dos repeticiones. Se pesaron las aves al inicio, antes y después de la recolección de heces. El período experimental tuvo una duración de 3 semanas. Las dietas, insumos y excretas fueron analizadas. Resultados: Se encontró diferencias significativas en la ganancia de peso y consumo de la dieta; mediante la prueba de Duncan se comprobó estadísticamente que la ganancia de peso en pollos alimentados con la dieta con el insumo aceite compuesto fue mayor que en pollos alimentados con la dieta referencial y éstos tuvieron un valor mayor que los pollos alimentados con dieta con el insumo aceite residual de fritura; esta misma relación se encontró con la variable consumo de la dieta. Conclusión: La energía metabolizable por el método de colección total en base húmeda, para el aceite compuesto fue de $9,989 \mathrm{kcal} / \mathrm{kg}$ y del aceite residual de fritura fue de $9,743 \mathrm{kcal} / \mathrm{kg}$, no habiendo diferencias significativas a la prueba de " $\mathrm{t}$ " entre los valores de energía metabolizable de aceite compuesto y aceite residual de fritura.

Palabras clave: Energía Metabolizable; Ácidos Grasos; Dieta; Ingesta.

\begin{abstract}
Objetive: Determine the metabolizable energy in chickens of the ingredients, composite oil and residual oil from frying, by means of the total collection method. Material and Methods: Three types of diets were used: referential diet, diet with the compound oil intake and diet with the residual oil intake of fried foods; Each diet was tested on 10 chickens with two repetitions. The birds were weighed at the beginning, before and after the collection of feces. The experimental period lasted 3 weeks. The diets, supplies and excreta were analyzed. Results: Significant differences were found in the weight gain and consumption of the diet; Duncan's test showed statistically that weight gain in chickens fed the diet with the compound oil input was greater than in chickens fed the reference diet and these had a higher value than chickens fed diet with the oil intake frying residual; this same relationship was found with the variable consumption of the diet. Conclusion: The energy metabolizable by the total collection method on a wet basis, for the composite oil was $9,989 \mathrm{kcal} / \mathrm{kg}$ and the residual frying oil was $9,743 \mathrm{kcal} / \mathrm{kg}$, there being no significant differences to the "t" test between the values of metabolizable energy of composite oil and residual frying oil.
\end{abstract}

Key words: Metabolizable Energy; Fatty acids; Diet; Ingestion.

\footnotetext{
${ }^{1}$ Docente de la Facultad de Ingeniería Agraria, Industria Alimentarias y Ambiental de la U.N.J.F.S.C. (Huacho - Perú). Email: djmcpe@yahoo.com

${ }^{2}$ Docente de la Facultad de Zootecnia. Universidad Nacional Agraria. La Molina (Lima - Perú).
} 


\section{INTRODUCCIÓN}

En nuestro país para elaborar aceites compuestos comestibles se emplea como materia prima aceites marinos comestibles que deben ser mezclados con aceite vegetal para el consumo humano, líquida a temperatura de $20^{\circ} \mathrm{C}$ olor y sabor característicos del producto desgomado, exento de sabores y olores extraños, no contener más de $0.1 \%$ de agua, no tener una acidez libre mayor de $0.35 \%$, un índice de peróxido de $5 \mathrm{meqO}_{2} / \mathrm{kg}$ grasa (INDECOPI, 1999) La proporción máximas aceptable de ambos aceites es del 50\% (Portocarrero, 1981). Los factores que afectan el valor energético de los aceites son: el tipo de grasa (Noy y Skalan, 1995; De Blas y Mateos, 1991); factores ligados al ave (Krogdahl, 1985); factores ligados a la dieta (De Blas y Mateos, 1991) $\mathrm{y}$ factores ligados al medio ambiente y presencia de contaminantes (Mateos, 1986)

La energía metabolizable es la proporción de energía del alimento que es disponible para el aves los diversos procesos corporales y es relativamente fácil de hallar, numerosas investigaciones reportan valores de energía metabolizable para diferentes aceites (Young y Artman, 1961; Lesson y Summers, 1997 y Seminario, 1971). El método directo para determinar la energía metabolizable consta de una dieta referencia en base a glucosa y dietas experimentales en las cuales una porción de la glucosa se reemplaza por el material a probar alimentando a las aves sin restricción (De Blas y Mateos, 1991). Los resultados son determinados comparando la energía que desaparece de la dieta problema y de la dieta referencia después de su pasaje a través del aparato digestivo de las aves (Rojas, 1979).

Uno de los principales usos que se les da a los aceites es en el proceso de frituras de alimentos, siendo estos rehusados reiteradamente para luego ser desechados. Este proceso de frituras donde se usa grandes cantidades de aceites se da generalmente en los negocios que se dedican a la venta de papas fritas, pollos fritos y otros preparados dejando como resultado cantidades apreciables de aceites que son eliminados. Se ha estimado que cerca del $50 \%$ de aceite se desecha (Ziller, 1996). El calentamiento prolongado a elevadas temperaturas en presencia de agua y oxigeno que contienen los alimentos provoca la oxidación del aceite dando lugar a una variedad de compuestos como: carbonilos volátiles, hidroxiácidos, cetoácidos y epoxiácidos que le confieren sabores desagradables y lo obscurecen (Fellows, 1994). Se ha encontrado en el aceite residual de fritura una disminución en ácidos grasos libres y valor peróxido y una disminución en índice de yodo como en los niveles de ácidos grasos poliinsaturados con respecto al aceite sin utilizar, presumiblemente por oxidación y polimerización (Nolen et al. 1967; Badui, 1984 y Hebbel, 1969)

Una disminución de la ingesta de alimentos o menor capacidad de absorción de la grasa, disminución del crecimiento (Nolen et al. 1967 y Cuesta et al. 1993) y aumento del tamaño del hígado y daños al riñón son algunos de los efectos adversos encontrados en animales alimentados con aceite de fritura. Sin embargo, se debe tener en cuenta que los estudios toxicológicos se hacen alimentando a los animales con grandes cantidades de aceite oxidado (Badui, 1984). Por otro lado, Lesson y Summers (1997) encontrarón un valor de energía metabolizable para grasa de restaurante de $8100 \mathrm{kcal} / \mathrm{kg}$ en pollos a los 21 días; mientras que Varela y Ruiz (1986) y Alexander, Chanin y Morón. (1983) determinaron pequeños cambios, pero sin diferencias significativas a la prueba "t", al comparar los valores en energía metabolizable de aceite sin utilizar y su aceite residual de fritura en ratas. El presente trabajo de investigación tiene como objetivo determinar la energía metabolizable del aceite compuesto y el aceite residual de fritura en pollos por el método de colección total.

\section{MATERIALES Y MÉTODOS}

La investigación se realizó en el Laboratorio de Evaluación Biológica en Aves, y los Laboratorios de Evaluación Nutricional de Alimentos perteneciente al Departamento Académico de Nutrición, así como también en la Planta Piloto del Programa de Alimentos de la Universidad Nacional Agraria la Molina,

En el presente experimento se utilizaron 90 pollos machos de la línea Ross de un día de edad, que posteriormente fueron randomizados y divididos en grupos de 10 que fue la unidad experimental. El tiempo de crianza fue de 21 días, siendo la primera semana una etapa pre experimental y las dos siguientes la etapa experimental, distribuidos en 10 días de adaptación a la dieta y los últimos 3 días de evaluaciones.

Las pruebas se efectuaron en jaulas metabólicas colectivas. Se utilizó una batería eléctrica de 5 pisos, cada piso con dos compartimentos. Cada jaula cuenta con calefacción automática, regulada por un termostato y controlado por un foco de 50 watts. Cada compartimiento está equipado con dos 
comederos laterales y un bebedero frontal y una bandeja para la colección de heces.

La composición de las dietas se muestra en la tabla 1. Se utilizó una dieta de referencia, con altos niveles de glucosa $(50 \%)$, en base a la cual se elaboró las dietas problemas sustituyendo la glucosa en un $10 \%$ por el aceite crudo y aceite de fritura, respectivamente.

Durante la primera semana todos los grupos recibieron la dieta de referencia, asignándoles las dietas experimentales a partir de la segunda semana de edad. El suministro del alimento y de agua fue ad libitum, renovando el agua diariamente.

Los productos a evaluar son el aceite compuesto de marca comercial "Cocinero" y su respectivo aceite residual de fritura, obtenido este último después de haber usado el aceite crudo por una semana (con reposición de aceite crudo para mantener el volumen de aceite en el recipiente constante), en fritura de pollos y papas del Restaurante "Don Narch" de Santa Felicia - La Molina. Se determinó la humedad, extracto etéreo, índice de yodo, índice de peróxido y ácidos grasos libres de los insumos a evaluar(A.O.A.C., 1980)

La energía metabolizable fue determinada después de 72 horas durante la tercera semana. Se tomó muestras representativas de los tres tipos de dietas y sus respectivas excretas para su determinación energética que se realizó mediante la Bomba calorimetrica Parr. Las excretas fueron colectadas por unidad experimental, se les seco y molió para su análisis. El consumo de alimentos y producción de excretas fueron controlados en los días de prueba.La energía metabolizable mediante la técnica de colección total se realizó según Hill y Anderson. (1960)

Se utilizó un diseño experimental completamente al azar para los datos de peso promedio y consumo promedio de los lotes a los 21 días de edad: distribuido en 3 dietas y 3 réplicas respectivamente. Posteriormente se realizó el análisis de variancia para determinar la presencia de diferencias significativas. Los resultados de energía metabolizable se reportan como el promedio más el error estándar. Se realizó la prueba de $t$ "para comparar tanto los valores de energía metabolizable del aceite compuesto y aceite residual de fritura (Calzada,1982)
Tabla 1. Composición porcentual en ingredientes de las dietas y valor nutritivo.

\begin{tabular}{|c|c|c|c|}
\hline \multicolumn{2}{|c|}{ Ingredientes } & $\begin{array}{r}\text { Dieta de } \\
\text { Referencia }\end{array}$ & $\begin{array}{c}\text { Dieta } \\
\text { Experimental }\end{array}$ \\
\hline \multicolumn{2}{|l|}{ Glucosa } & 50 & 40 \\
\hline \multicolumn{2}{|c|}{ Harina de Soya } & 40,87 & 40,87 \\
\hline \multicolumn{2}{|c|}{ Harina de Pescado,65 } & 3,36 & 3,36 \\
\hline \multicolumn{2}{|c|}{ Harina de Huesos } & 1,96 & 1,96 \\
\hline \multicolumn{2}{|c|}{ Carbonato de Calcio } & 1,25 & 1,25 \\
\hline \multicolumn{2}{|c|}{ Aceite compuesto } & 1,92 & 1,92 \\
\hline \multicolumn{2}{|l|}{ Sal común } & 0,36 & 0,36 \\
\hline \multicolumn{2}{|c|}{ DL Metionina } & 0,21 & 0,21 \\
\hline \multicolumn{2}{|c|}{ Cloruro de Colina, 60} & 0,05 & 0,05 \\
\hline \multicolumn{2}{|c|}{ Premezcla de vitaminas y minerales } & 0,1 & 0,1 \\
\hline \multicolumn{2}{|c|}{ Aceite problema } & - & 10 \\
\hline & Total & 100 & 100 \\
\hline \multicolumn{4}{|c|}{ Valor Nutritivo } \\
\hline \multicolumn{2}{|l|}{$\mathrm{EM} \mathrm{Mcal} / \mathrm{kg}$} & 3,060 & 3,480 \\
\hline Proteína & $\%$ & 21,08 & 21,13 \\
\hline Lisina & $\%$ & 1,29 & 1,29 \\
\hline Metionina & $\%$ & 0,48 & 0,48 \\
\hline Met - Cis & $\%$ & 0,77 & 0,77 \\
\hline Calcio & $\%$ & 0,8 & 0,8 \\
\hline Fósforo disp. & $\%$ & 0,4 & 0,4 \\
\hline Sodio & $\%$ & 0,22 & 0,22 \\
\hline
\end{tabular}

\section{RESULTADOS Y DISCUSIÓN}

Los pollos tuvieron un adecuado comportamiento productivo con todas las dietas y entre repeticiones, con lo cual sé aseguró que no existió condiciones desfavorables o falta de uniformidad del medio ambiente que podrían haber afectado la determinación de energía metabolizable.

Los resultados sobre ganancia de peso y consumo de alimentos a los 21 días se reportan en la tabla 2. Los pollos alimentados con la dieta conteniendo aceite compuesto mostraron diferencias significativas en ganancia de peso y consumo de alimentos respecto a los pollos alimentados con la dieta referencia, obteniendo los primeros una mayor ganancia de peso y un mayor consumo de alimentos; este resultado corrobora la afirmación de Zumbado(1990), quien menciona que al usar aceite en la dieta, hay una mayor ganancia de peso y aumento en el consumo de la dieta debido a la mayor densidad energética en la dieta, al aporte de ácidos grasos esenciales que hacen a los alimentos más apetitosos fomentando su consumo, 
disminuyendo la velocidad de transito de la dieta por el intestino para una mejor y mayor absorción; teniendo por lo tanto un mejor comportamiento productivo. Por otro lado comparando los valores de ganancia de peso y consumo de alimentos de los pollos alimentados con dieta con aceite residual de fritura y pollos alimentados con la dieta referencia, se encontró que hubo diferencias significativa ; estos últimos pollos tenían mayores valores en las variables mencionadas, este resultado tiene cierta similitud con los valores hallados por Pilares (1997), para subproductos de aceite pescado donde había una mayor concentración de ácidos grasos libres, menor grado de insaturación de sus ácidos grasos, como se da en el aceite residual de fritura, que influirán negativamente en la formación de micelas en el lumen intestinal para su absorción; además que los productos formados en la fritura (polímeros, peróxidos, etc.) son inabsorbibles y hacen que la velocidad de tránsito de la dieta sea mayor, perjudicando la absorción de los otros nutrientes (Squires, Valdez y Lesson., 1991).

Tabla 2. Ganancia de peso (g) y consumo de alimento(o) nromedio nor dieto

\begin{tabular}{lcc} 
Dieta & $\begin{array}{c}\text { Ganancia de } \\
\text { peso }(\mathrm{g})^{*}\end{array}$ & $\begin{array}{c}\text { Consumo de } \\
\text { alimentos }(\mathrm{g})^{*}\end{array}$ \\
\hline *Referencia & 264 & 385 \\
Con aceite compuesto & 285 & 407 \\
Con aceite residual de fritura & 249 & 369 \\
\hline
\end{tabular}

\footnotetext{
*Al análisis de variancia y prueba de Duncan se demostró que hay diferencias significativas entre todas las dietas
}

Asimismo, los pollos alimentados con dieta conteniendo aceite compuesto tuvieron diferencias significativas en ganancia de peso y consumo de alimentos con los pollos alimentados con dieta conteniendo aceite residual de fritura; mostrando los pollos alimentado con dieta conteniendo aceite sin utilizar una mayor ganancia de peso y mayor consumo de alimento; estos resultados corroboran los hallados por Cuesta et al. (1993); Nolen et al. (1967). Esto se debe a que en el aceite residual de fritura hay un aumento en la cantidad de ácidos grasos libres, una disminución en la relación ácido graso insaturado /ácido graso saturado, disminución del índice de yodo y aumento en el valor peróxido respecto al aceite crudo, como se muestra en la tabla 4, además en el aceite de fritura se tiene la presencia de hidrocarburos, cetonas, aldheidos, dímeros y otro compuestos inabsorbibles que se forman debido al calentamiento del aceite crudo a partir de los ácidos grasos saturados e insaturados que éste contiene.
Los resultados de la energía metabolizable tanto del aceite compuesto como el aceite residual de fritura son mostrados en la tabla 3. Comparando los valores de energía metabolizable del aceite compuesto y su aceite residual de fritura, se observa que los valores son mayores para el aceite compuesto sin embargo no hay diferencias estadisticamente significativas Los valores son $9989 \mathrm{kcal} / \mathrm{kg}$ y $97433 \mathrm{kcal} / \mathrm{kg}$ respectivamente, se observa que la energía metabolizable del aceite compuesto es mayor que la energía metabolizable de su aceite residual en un 2,71\%. Estos resultados están de acuerdo con Alexander et. al. (1983), quien encontró en que no había diferencias entre las energías metabolizables de aceite de maíz sin utilizar y su respectivo aceite residual de fritura obtenida a partir de fritura de pollos a profundidad bajo presión. Al respecto Varela y Ruiz (1986) evaluando aceite de soya, aceite de oliva y grasa sólida que fueron usadas para freír papas, determinó que no había diferencias significativas en los valores de energía digestible, con respecto a sus aceites sin utilizar. Ambos trabajos de investigación se realizaron en ratas.

El valor de la energía metabolizable del aceite compuesto por el método de colección total fue de $9989 \mathrm{kcal} / \mathrm{kg}$. Al respecto para determinaciones de energía metabolizable, en pollos, de aceites marinos se reporta que, se encontró que el aceite semirrefinado de anchoveta tuvo un valor de energía metabolizable de $8550 \mathrm{kcal} / \mathrm{kg}$, (Seminario, 1971) mientras que Guevara (1997) concluyó que un aceite acidulado y estabilizado de pescado como producto final tuvo de energía metabolizable el valor de $8658 \mathrm{kcal} / \mathrm{kg}$ empleando para ello la técnica de colección total y remplazando a la fuente energética glucosa en un 10\%. Al respecto Lesson y Summers (1997) reporta que la energía metabolizable de aceite de pescado fue de $8600 \mathrm{kcal} / \mathrm{kg}$ a la edad de 3 semanas para luego elevarse a $9000 \mathrm{Kcal} / \mathrm{kg}$ en pollos de más edad.

Por otro lado para determinaciones con aceites vegetales, Young y Artman (1960) encontraron que los valores de energía metabolizable, en pollos , para aceite de soya y aceite de maíz son de 9260 y $8440 \mathrm{kcal} / \mathrm{kg}$ respectivamente cuando representaron el $15 \%$ de la dieta mientras que Lesson y Summers (1997) refieren que el valor para el aceite vegetal es $8800 \mathrm{kcal} / \mathrm{kg}$ a las tres semanas de edad para luego elevarse a $9000 \mathrm{kcal} / \mathrm{kg}$ cuando son de más edad, por su parte Vint (1991) reporta que el aceite de semilla de algodón tiene 8800 
$\mathrm{kcal} / \mathrm{kg}$, y Wiseman et al. (1992) reportan para el aceite de girasol un valor de $8963 \mathrm{kcal} / \mathrm{kg}$ aunque Dale y Fuller, (1982) reportan un valor 10780 $\mathrm{kcal} / \mathrm{kg}$ de energía metabolizable para el aceite de maíz cuando su nivel de inclusión en la dieta fue del 2,5\%. Para determinaciones con aceite compuesto (mezcla de grasa vegetal y animal) se reportan los valores de $8200 \mathrm{kcal} / \mathrm{kg}$ (Vint, 1991) y $8500 \mathrm{kcal} / \mathrm{kg}$ obtenidos en pollos de 3 semanas de edad y $8600 \mathrm{kcal} / \mathrm{kg}$ para pollos mayores de tres semanas de edad (Lesson y Summers, 1997).

Tabla 3. Contenido de energía metabolizable del aceite compuesto crudo y el aceite residual de fritura

\begin{tabular}{lccc}
\hline $\begin{array}{l}\text { Energía } \\
\mathrm{kcal} / \mathrm{kg}\end{array}$ & $\begin{array}{c}\text { Dieta de } \\
\text { Referencia }\end{array}$ & $\begin{array}{c}\text { Dieta con aceite } \\
\text { compuesto }\end{array}$ & $\begin{array}{c}\text { Dieta con aceite de } \\
\text { fritura }\end{array}$ \\
\hline Método colección total & & & \\
E.B. Dieta & 4197 & 4826 & 4703 \\
E.B. Excreta & 3561 & 3816 & 3947 \\
Excreta (g M.S./día) & 22,85 & 22,7 & 18,83 \\
Consumo (g M.S./día) & 86,57 & 92,66 & 84,13 \\
Excreta/Consumo & 0,26 & 0,24 & 0,21 \\
E.M. Referencia & 3,25 & 3,88 & 3,86 \\
E.M. Insumo Graso* & & 9989 & 9743 \\
\hline
\end{tabular}

*E.M. $=3.65-($ E.M. Referencia -E.M. Grasa) $/ 0.10$ No se encuentran diferencias entre formas de grasa.

Comparando estos valores de energía metabolizable de distintos aceites con el resultado de energía metabolizable obtenido del aceite compuesto, observamos que nuestro valor promedio es generalmente mayor, esta variación se puede deber a la composición del aceite compuesto comercial usado (mezcla de aceite de pescado y vegetal), raza y edad del ave, factores del medio ambiente, composición y consumo de la dieta, nivel de insaturación, relación entre ácidos grasos insaturados y saturados. Se ha demostrado además que el valor de la energía metabolizable aparente de las grasas varía con la naturaleza de la dieta referencia (De Blas y Mateos, 1991)

El valor de energía metabolizable del aceite residual de fritura por el método de colección total obtenido es de $9743 \mathrm{kcal} / \mathrm{kg}$. Comparando con los resultados que nos reporta Lesson y Summers (1997) que fue de $8100 \mathrm{kcal} / \mathrm{kg}$ para pollos a las tres semanas de edad y $8900 \mathrm{kcal} / \mathrm{kg}$ para pollos mayores de tres semanas de edad; se puede comprobar que nuestro valor obtenido es mayor, esta diferencia se debe a la calidad del aceite crudo expuesto al proceso de fritura, al tipo de alimento a freír, a la temperatura y tiempo de exposición en la freidora, al área superficial del aceite expuesta al oxigeno del aire, a la humedad liberada por el alimento y a la velocidad de reposición del aceite que determinaran los niveles de oxidación, hidrólisis y posterior polimerización de los aceites como menciona Ziller (1996).

El valor energético del aceite depende de la naturaleza de la estructura química de los ácidos grasos presentes, que se traduce en tres índices de calidad; el índice de yodo, el índice de peróxido y el índice de ácidos grasos libres cuyos resultados se muestran en la tabla 4. En los aceites sin utilizar se encuentra un mayor valor del índice de yodo respecto a los aceites residuales de fritura, que refleja el grado de insaturación de la estructura química. Al respecto Hemans et al. (1973) y Hebbel et al., (1969) encontraron que los ácidos grasos polinsaturados se degradaban para dar ácidos grasos monoinsaturados y saturados aumentando la concentración de estos compuestos conforme aumentaba el tiempo de fritura, ambos autores encontraron que el índice de yodo disminuía conforme sucedían estos cambios. Alexander et al. (1983) nos menciona que este cambio es más severo a nivel comercial. Por otro lado, De Blas y Mateos (1991) refieren que los ácidos grasos poliinsaturados son mejor absorbidos por las aves que los monoinsaturados y éstos que los saturados.

El índice de peróxido es mayor en aceite residual de fritura que en aceite sin utilizar, Badui (1985) confirma este resultado. Sin embargo, Yepez et al. (1990) refieren que este indicador no sería adecuado para ver la calidad del aceite de fritura y que tendría que complementarse con otros indicadores por cuanto los peróxidos se descomponen a compuestos carbonílicos y esto se acentúa cuando aumenta la temperatura. Por otro lado, se ha observado una relación directa entre un aumento del índice de peróxido y un menor tiempo de permanencia en el tracto intestinal disminuyendo su digestibilidad (Squires et al. 1991).

Tabla 4. Análisis químicos de los insumos grasos

\begin{tabular}{lccc}
\hline & $\begin{array}{c}\text { Aceite } \\
\text { compuesto }\end{array}$ & $\begin{array}{c}\text { Aceite resudial de } \\
\text { fritura }\end{array}$ & INDECOPI \\
\hline Extracto Etéreo (\%) & 100 & 99 & $100^{*}$ \\
Humedad (\%) & 0,00 & 0,8 & $<0,10$ \\
Índice de Peróxido (meq O $/ \mathrm{kg}$ ) & 0,96 & 2,32 & $<0,50$ \\
Índice de Iodo (g. de yodo $/ 100 \mathrm{~g})$ & 110,67 & 106,6 & no reporte \\
Ácidos grasos (\%) & 0,23 & 0,68 & $<0,3$ \\
\hline
\end{tabular}

Fuente: *INDECOPI (1999): Norma de Calidad del Aceite Compuesto 
El porcentaje de los ácidos grasos libres presentes en el aceite sin utilizar es menor que el presentado por el aceite residual de fritura que va de 0,23 a $0,68 \%$ respectivamente, estos resultados son similares a los encontrados por Nolen et al. (1967), Hemans et al. (1973) y Badui (1984). Por otro lado, De Blas y Mateos (1991) refieren que es preferible que las grasas tengan una menor cantidad de ácidos grasos libres por cuanto ello influye en la formación de micelas que son el medio por el cual las grasas son absorbidas.

El contenido de extracto etéreo, mostrado en la tabla 4, del aceite compuesto fue de $100 \%$, este valor es similar al reportado para el aceite de oliva $(99,8 \%)$ por Varela y Ruiz (1986) y para el aceite de algodón (100\%) por Hemans et al. (1973), igualmente éstos últimos refieren que el extracto etéreo para el aceite caliente de algodón es de $99,8 \%$ teniendo una mínima diferencia con el valor determinado para el aceite de fritura que fue de $99 \%$. Comparando los valores de extracto etéreo del aceite compuesto con el aceite residual de fritura se observa que la diferencia es mínima, esto es corroborado por Hemans et al. (1973), quien observo lo mismo entre el aceite de algodón sin utilizar y su aceite caliente, aseverando que la variación esta en su composición de ácidos grasos saturados, mono y poli insaturados.

INDECOPI (1999) menciona que el aceite

\section{REFERENCIA BIBLIOGRÁFICA}

Alexander, J. ; B.E. Chanin \& E. T. Moran 1983 Nutritional effects of fresh, laboratory heated, and pressure deep - fry fats. Journal of Food Science. Vol.48 p.1289-1292

Association of Oficial Analytical Chemists(A.O.A.C.) .1980 Official methods of analysis Thirteeth Edition .Arlincton USA. pag. 134-138

Badui ,S. 1984 Química de los Alimentos. Edit Alambra a México D.F.

Calzada, B. 1982 Métodos Estadísticos de Investigación Vol 1 . 680paginas. Ediciones Universidad Nacional Agraria. Lima- Peru

Cuesta ,C. ; F. Sanchez- Muñiz \& S. Lopez- Varela. 1993 Alteración termo oxidativa en un aceite de girasol utilizado en 75 frituras de patatas; efecto de su inclusión en dietas sobre crecimiento e ingesta en ratas . Revista Grasas y Aceites. Jul-Oct. Vol 44(4-5) p. 263- 269. España.

Fellows ,P. 1994 Tecnología del Procesado de los Alimentos . Edit. Acribia S.A. Zaragoza España

Dale, N. \& L. Fuller . 1982 True metabolizable comestible compuesto debe contener como máximo de humedad $0,1 \%$. Young y Artman (1961) encontraron en aceite de soya, maíz y mezcla de grasa animal y vegetal el valor de $0,07 \%$, siendo estos valores similares al encontrado. El porcentaje de humedad refiere Hemans et al. (1973) para el aceite de algodón calentado fue menor de $1 \%$ mientras que Sung, (2000) menciona que el valor de humedad para el aceite residual de fritura esta entre 0,5 y $1,5 \%$ corroborando el valor encontrado $(0,8 \%)$; habiendo entonces una ligera diferencia entre la humedad del aceite compuesto y el aceite residual de fritura determinado, que se muestra en la tabla 4. Un aumento de la humedad diluye el contenido de energía, facilita la corrosión de accesorios con que se manejan las grasas además en su determinación ocurre que el agua se deposita en el fondo y a una previa agitación su distribución es no homogénea haciendo que la muestra de aceite tomada no sea representativa (Sung, 2000)

\section{CONCLUSIÓN}

Bajo las condiciones experimentales del presente estudio, se llegó a la siguiente conclusión: La energía metabolizable en base húmeda por el método de colección total para el aceite compuesto fue de $9989 \mathrm{kcal} / \mathrm{kg}$ y para el aceite residual de fritura fue de $9659 \mathrm{kcal} / \mathrm{kg}$, siendo estos valores estadísticamente similares.

energy of fats at low dietary inclusion Poultry Science 61: 2415 - 2421. U.S.A.

De Blas, C. \& G. Mateos. 1991 Nutrición y Alimentación de Gallinas Ponedoras .Ministerio de Agricultura y Pesca. Madrid. España.

Guevara, V. 1997 Determinación de la energía metabolizable de enermas para aves. Revista Mundo Avicola y Porcino $N^{\circ} 21$ Ene- Feb.

Hebbel, S.; L.. Masson \& R. Rybertt.. 1969. Estudio de la acción del calor sobre algunos aceites vegetales usados en la elaboración de papas . Rev. Agroquímica y tecnología Alimentaria Vol $9 \# 3$. España

Hemans, C ., F. Kummerow \& E. Perkins. 1973. Influence of protein and vitamin levels on the nutritional value of heated fats for rats. J.Nutr. 103: $1665-1672$

Hill, F \& D. Anderson . 1960 Comparasion of metabolizable energy and productive energy determinations with griwing chicks . J . Nutrition 64: 587-603

Instituto de Defensa del consumidor y de la propiedad 
Intelectual (INDECOPI) 1999 . Norma del ITINTEC 209. 07 ${ }^{\text {a }}$ Aceites Compuestos Comestibles. Lima Perú

Krojdalh, A. 1985 Digestión and absorption of lipids in poultry. J. Nutritión 115: 675 - 685

Lesson, S \& J.D. Summers. 1997 Commercial Poultry Production.2nd. Edition. University Books, Guelph. Ontario Canada.

Mateos, G. 1986 Dietary fat as an antiheat - factor in Poultry. 4to Congreso Mundial de Alimentación Animal. Madrid. 180pag.

Nolen,A. ; J. Craig, \& R. Artman. 1967 Longterm rat feeding study with used frying fats. J. Nutrition $93: 337-348$.

Noy J. \& D. Skalan 1995 Digestion and absorbption in the young chick . Poultry Science : 74 : 366-373

Pilares, D. 1997 Determinación de la Energía Metabolizable de la Grasa Acida, Estearina y Acidos Grasos de Pescado en Pollos de Carne. Tesis de Grado Mg. Sc. Escuela de Post Grado Universidad Nacional Agraria La Molina .Lima-Perú

Portocarrero, R. 1981 Estudio Preliminar para la Determinación Cuantitativa de Aceite de Pescado en Aceite Compuesto. Tesis Ing Pesquero. Universidad Nacional Agraria. Lima - Perú

Rojas, S. 1978 Nutrición Animal Aplicada . Departamento de Nutrición. Universidad Nacional Agraria. La Molina. Lima- Perú

Seminario, R. 1971 Estudio Comparativo del Aceite de Pescado Hidrogenizado con el Sebo de Vacuno, Aceite de Soya y Aceite de Pescado como Fuente de Energía en Raciones de Aves.
Tesis Ing. Zootecnista . Universidad Nacional Agraria La Molina. Perú.

Squires , E. ; J. Valdez \& S. Lesson. 1991 Research notes : utility of the thiobarbituric acid test in the determination of the quality of fats and oil in feed. Poultry Science . 70: 180-183.

Sung, I. 2000 Nutrición y Salud . Editorial Isabel Lima-Perú . pag. 35

Varela, G. \& B. Ruiz. 1986 Somme effects of deep frying on dietary fat intake. Nutrtition Reviews Vol.50 No 9

Vint, L. 1991 La grasa en las raciones para pollos y reposición y ponedoras. Rev. Avicultura Profesional Vol $1 \# 1$

Wiseman ,J. ; B.K. Edmonds \& N. Shepperson. 1992 .The apparent metabolizable energy sunflower oil and sunflower acid oil for broiler chickens. Animal Feeed Sci Tech . 36:41-51

Yepez,F.;R. Guillen.; \& M.Guzman. 1990. Estudio de estabilidad de aceite de oliva, sometido a un método de autooxidación acelerada por calentamiento. Anales- de- Bromatología (Spain).(Jan 1990).v. 41(2) p. 223-232.

Young, R. \& R. Artman . 1961 The energy value of fats and fatty acids for chicks . Departament of Poultry Husbandry, Cornell University , Ithaca, New York.

Ziller, S. 1996 Grasas y Aceites Alimentarios. Edit. Acribia. Zaragoza. España.

Zumbado, M. 1990 Utilización de productos de la palma africana en la alimentación aviar. En Rev. Avicultura Profesional. Vol 7\#4 